Research Paper

\title{
Weekly versus triweekly cisplatin plus intensity-modulated radiotherapy in locally advanced nasopharyngeal carcinoma: A propensity score analysis with a large cohort
}

\author{
Qian Zhu ${ }^{1,2^{*}}$, Hao $\mathrm{Hu}^{3 *}$, Lin-Quan Tang1,4, Rui You ${ }^{1,4}$, Jing-Jing Zhao, ${ }^{1,2}$, De-Sheng Weng ${ }^{1,2}$, Qiu-Zhong \\ Pan $^{1,2}$, Chang-Long Chen ${ }^{1,2}$, Zi-Qi Zhou ${ }^{1,2}$, Yan Tang ${ }^{1,2}$, Jian-Chuan Xia ${ }^{1,2}{ }^{\bowtie}$ \\ 1. State Key Laboratory of Oncology in South China, Collaborative Innovation Center for Cancer Medicine, Guangdong Key Laboratory of Nasopharyngeal \\ Carcinoma Diagnosis and Therapy, Sun Yat-sen University Cancer Center, Guangzhou 510060, P. R. China \\ 2. Department of Biotherapy, Sun Yat-Sen University Cancer Center, Guangzhou 510060, People's Republic of China \\ 3. Department of Surgery, Medical College of Nanchang University, Nanchang 330006, People's Republic of China \\ 4. Department of Nasopharyngeal Carcinoma, Sun Yat-Sen University Cancer Center, Guangzhou 510060, People's Republic of China \\ "These authors contributed equally to this work.
}

$\square$ Corresponding author: Prof. Jian-Chuan Xia, Department of Biotherapy, Sun Yat-Sen University Cancer Center, 651 Dongfeng Dong Road, Guangzhou 510060, People's Republic of China. (E-mail: xiajch@mail.sysu.edu.cn)

(c) Ivyspring International Publisher. This is an open access article distributed under the terms of the Creative Commons Attribution (CC BY-NC) license (https://creativecommons.org/licenses/by-nc/4.0/). See http://ivyspring.com/terms for full terms and conditions.

Received: 2018.03.16; Accepted: 2018.07.23; Published: 2018.09.08

\begin{abstract}
Purpose: To directly compare the efficacy and acute toxicities of intensity-modulated radiotherapy (IMRT) concurrent with weekly cisplatin $\left(40 \mathrm{mg} / \mathrm{m}^{2}\right)$ to high-dose concurrent cisplatin $\left(100 \mathrm{mg} / \mathrm{m}^{2}\right)$ at three-week intervals.

Materials and Methods: A total of 3,799 patients diagnosed with locally advanced nasopharyngeal carcinoma (NPC) at Sun Yat-Sen University Cancer Center between January 2010 and December 2013 were retrospectively reviewed. Propensity score analysis was conducted to balance the baseline characteristics between the groups, which allowed us to draw reliable conclusions. The efficacy and safety profiles were then assessed in the well-balanced large cohort.

Results: The risk of distant metastasis was lower among the patients treated with weekly concurrent cisplatin than among those treated with the triweekly regimen (hazard ratio [HR], 0.45; $\mathrm{P}=.028$ ). However, the disease-free survival, loco-regional relapse-free survival and overall survival rates were similar. The weekly group showed significantly higher rates of grade 3-4 thrombocytopenia, but lower rates of grade 3-4 mucositis, nausea and vomiting than the triweekly group.

Conclusion: IMRT concurrent with a weekly cisplatin regimen was associated with significantly improved distant metastasis-free survival in locally advanced NPC. Differences in the selected acute toxicities between the weekly and triweekly concurrent cisplatin regimens were noted.
\end{abstract}

Key words: nasopharyngeal carcinoma; intensity-modulated radiotherapy; cisplatin; concurrent chemoradiotherapy; survival outcome; adverse events.

\section{Introduction}

Nasopharyngeal carcinoma (NPC) is highly prevalent in eastern Asia, with the highest incidence reported in the southern provinces of China [1]. Due to its anatomical location and non-specific symptoms, early diagnosis and treatment of NPC are difficult [2,
3]. According to the 7th Edition of the American Joint Commission on Cancer Staging System, $60-70 \%$ of NPC patients present with locally advanced (stage III-IVB) disease [4], which carries a higher risk of loco-regional recurrence and distant metastasis [5]. 
For those relapsed with distant metastasis, the prognosis is poor, with a median survival ranging from 5 to 11 months [6-8].

Numerous randomized clinical trials have demonstrated that concurrent chemo-radiotherapy confers a survival benefit in locally advanced NPC [9-12]. Cisplatin-based concomitant chemo-radiotherapy (CDDP) has been selected as the standard treatment regimen for patients with locally advanced stage NPC [13]. The 2013 National Comprehensive Cancer Network (NCCN) guidelines recommend that concurrent cisplatin be delivered at an intermediate dose weekly $\left(40 \mathrm{mg} / \mathrm{m}^{2}\right)$ or a high dose $\left(100 \mathrm{mg} / \mathrm{m}^{2}\right)$ at three-week intervals for stage III-IVB NPC [14](14)(14)(14). Three studies compared these regimens and reported comparable survival outcomes (overall survival and disease-free survival) based on small sample sizes [15-17]. However, the clinical characteristics between the groups were unevenly distributed, and patients treated with 3-dimensional conformal radiotherapy were also included, which might confound the findings.

With these regimens, excellent local control can be realized with recent technical improvements in radiotherapy delivery, such as intensity-modulated radiation therapy (IMRT), and distant metastasis is now the main cause of treatment failure [18]. Therefore, the distant metastasis-free survival outcome is the crucial evaluation indicator of treatment efficiency, especially in locally advanced NPC patients.

The present study conducted propensity score analysis to compare the efficacy and acute toxicities between different CDDP treatment schedules (weekly vs. triweekly) in a large cohort. Moreover, we selected distant metastasis-free survival (DMFS) and loco-regional relapse-free survival (LRRFS), apart from overall survival (OS) and disease-free survival (DFS), as the evaluation indicators of efficacy. Our results provide evidence for choosing the appropriate CDDP treatment strategy for locally advanced NPC patients and will inform the design of future prospective studies.

\section{Materials and Methods}

\section{Patient selection and study design}

A total of 3,799 patients diagnosed with locally advanced NPC at Sun Yat-Sen University Cancer Center between January 2010 and December 2013 were retrospectively reviewed. Given the long time span, tumour grade and stage were restaged according to the International Union Against Cancer (UICC) Staging System (7th edition, 2011). Prior patient consent and approval from the Sun Yat-sen
University Cancer Center Institutional Review Board were obtained for the use of clinical data. The inclusion criteria were defined as follows: (a) histologically proven NPC; (b) clinically diagnosed as stage III-IVb disease; and (c) treated with cisplatin chemotherapy concurrent with IMRT. The exclusion criteria were as follows: (a) concurrent malignant disease; and (b) the use of adjuvant chemotherapy or additional concurrent targeted therapy.

Finally, 926 patients were evaluated in our study after applying the inclusion and exclusion criteria. We further used propensity scores to adjust for age, sex, Karnofsky performance status score (KPS), tumour stage ( $\mathrm{T}$ classification), node stage ( $\mathrm{N}$ classification) and clinical stage and then created a well-balanced cohort by matching each patient who underwent weekly cisplatin concurrent chemotherapy with no more than three patients who underwent IMRT plus triweekly cisplatin concurrent chemotherapy. From this stratification process, we selected a total of 859 patients, comprising 225 patients in the weekly CDDP group and 634 patients in the triweekly CDDP group (Table 1). Then, we conducted case-matched comparisons of the treatment efficacy and safety in this well-balanced large cohort. The flow diagram of this study design is shown in Figure 1. Moreover, we conducted multivariate and subgroup analyses of all 3,799 cases. The key raw data of this study has been validated by uploading onto the Research Data Deposit public platform (www.researchdata.org.cn) at Sun Yat-sen University Cancer Center.

\section{Treatment}

The radiation technology used in the present study was IMRT. All patients were treated with radiotherapy 5 days per week in once-daily fractions. The details of the prescribed radiation dose used at SYSUCC have been described in previous studies [16, 19]. Radiotherapy quality assurance (QA) was performed before radiation treatment for all the NPC patients. CDDP was initiated on the first day of radiotherapy, and the choice of cisplatin every three weeks $\left(100 \mathrm{mg} / \mathrm{m}^{2}\right)$ on days 1,22 , and 43 during radiotherapy or weekly CDDP $\left(40 \mathrm{mg} / \mathrm{m}^{2}\right)$ was based on the oncologists' opinions.

\section{Evaluation and follow-up}

Patients were examined weekly during the course of CDDP. Chemotherapy-related acute toxicities were graded according to the Common Terminology Criteria for Adverse Events (CTCAE) version 4.0. Radiotherapy-related toxic effects were evaluated based on the Late Radiation Morbidity Scoring Criteria of the Radiation Therapy Oncology Group. Acute toxicities were defined as those 
occurring either during the course of IMRT or within 90 days of its completion.

The follow-up period was the duration from the date of entry into the study to the day of death or the day of the last follow-up (10 October, 2017). After treatment, patients were observed at least once every 3 months during the first 3 years and then every 6 months thereafter until death. At every follow-up, we assessed disease status with complete nasopharyngoscopy, magnetic resonance imaging (MRI) of the head and neck, chest radiography, abdominal sonography, whole-body bone scan or positron emission tomography-computed tomography (PET-CT). DFS was defined as the date of pathological diagnosis to the date of the first recurrence/distant metastasis at any site, death from any cause, or the date of last follow-up visit. DMFS was defined as the date of diagnosis to the date of distant metastasis at any site. LRRFS was defined as the date of diagnosis to the date of loco-regional relapse. OS was calculated as the length of time between the date of initial pathological diagnosis and the date of all death events or last follow-up.

\section{Statistical analysis}

Statistical analyses were performed using SPSS version 22.0 software. Categorical variables and acute toxicities were compared using the $\chi^{2}$ or Fisher's exact test. Continuous variables were analysed using the Mann-Whitney $U$-test. The survival curves were constructed using the Kaplan-Meier method and analysed using the log-rank test. Prognostic factors for survival outcome were analysed with univariate and multivariate Cox regression analyses. The results are presented as hazard ratios (HR) and 95\% confidence intervals $(\mathrm{CI})$. $\mathrm{HR}>1$ indicates an elevated risk relative to the reference variable. An interaction term between the treatment regimen status and the independent prognostic factors was added to test the effect of their interaction. In all analyses, statistical significance was set at a $P$ value less than 0.05 (two-tailed).

\section{Results}

\section{Patient characteristics and compliance}

The regimen of triweekly CDDP (699) was more frequently used compared with weekly CDDP (227) in our cancer centre (Figure 1). After propensity score matching (PSM), 634 patients were included in the triweekly group and 225 patients in the weekly group. The baseline demographic and clinical characteristics were well balanced between the two groups (Table 1). All the patients (859) completed IMRT at the recommended dose.

For both groups, the chemotherapy cycles were more than one. All of the patients received $100 \mathrm{mg} / \mathrm{m}^{2}$ in the triweekly group and $40 \mathrm{mg} / \mathrm{m}^{2}$ cisplatin in the weekly group. The mean cumulative dose of cisplatin was similar between the weekly and triweekly groups $\left(229.2 \mathrm{mg} / \mathrm{m}^{2}\right.$ vs. $\left.228.0 \mathrm{mg} / \mathrm{m}^{2}\right)$. In the weekly group, the majority $(206 / 225,92 \%)$ of patients received at least five chemotherapy cycles, including 39/225 (17\%) patients with seven cycles of CDDP (IQR 5-6) (Supplementary Figure 1A). In the triweekly group, $619 / 634(97 \%)$ patients received at least two cycles of cisplatin chemotherapy, including 192/634 (30\%) patients who completed three cycles of concurrent chemotherapy (IQR 2-3) (Supplementary Figure 1B).

Table 1. Baseline characteristics of the unmatched and matched groups

\begin{tabular}{|c|c|c|c|c|c|c|}
\hline \multirow[t]{2}{*}{ Characteristics } & \multicolumn{3}{|c|}{ Before Matching $(\mathrm{N}=926)$} & \multicolumn{3}{|c|}{ After Matching $(\mathrm{N}=859)$} \\
\hline & Weekly $(\mathrm{N}=227)$ & Triweekly $(\mathrm{N}=699)$ & $P \pi$ & Weekly $(\mathrm{N}=225)$ & Triweekly(N=634) & $P \uparrow$ \\
\hline Age, years & $45.1(15-72)$ & $45.3(18-74)$ & $0.838 \&$ & $45.2(15-72)$ & $45.0(18-74)$ & $0.830^{8}$ \\
\hline \multicolumn{7}{|l|}{ Sex } \\
\hline Male & $171(75.3 \%)$ & $519(25.8 \%)$ & 0.745 & $170(75.6 \%)$ & $475(74.9 \%)$ & 0.850 \\
\hline Female & $56(24.7 \%)$ & $180(74.2 \%)$ & & $55(24.4 \%)$ & $159(25.1 \%)$ & \\
\hline \multicolumn{7}{|l|}{ KPS } \\
\hline $90-100$ & $222(97.8 \%)$ & $690(98.7 \%)$ & 0.349 & $222(98.7 \%)$ & $630(99.4 \%)$ & 0.386 \\
\hline $70-80$ & $5(2.2 \%)$ & $9(1.3 \%)$ & & $3(1.3 \%)$ & $4(0.6 \%)$ & \\
\hline \multicolumn{7}{|l|}{ T classification } \\
\hline $\mathrm{T} 1$ & $7(3.1 \%)$ & $15(2.1 \%)$ & 0.336 & $7(3.1 \%)$ & $15(2.3 \%)$ & 0.802 \\
\hline $\mathrm{T} 2$ & $27(11.9 \%)$ & $72(10.3 \%)$ & & $26(11.6 \%)$ & $62(9.8 \%)$ & \\
\hline T3 & $163(71.8 \%)$ & $487(69.7 \%)$ & & $162(72.0 \%)$ & $469(74.0 \%)$ & \\
\hline $\mathrm{T} 4$ & $30(13.2 \%)$ & $125(17.9 \%)$ & & $30(13.3 \%)$ & $88(13.9 \%)$ & \\
\hline \multicolumn{7}{|l|}{$\mathrm{N}$ classification } \\
\hline N0 & $22(9.7 \%)$ & $68(9.7 \%)$ & 0.320 & $22(9.8 \%)$ & $61(9.6 \%)$ & 0.494 \\
\hline N1 & $103(45.4 \%)$ & $342(48.9 \%)$ & & $103(45.8 \%)$ & $320(40.5 \%)$ & \\
\hline $\mathrm{N} 2$ & $91(40.1 \%)$ & $239(34.2 \%)$ & & $90(40.0 \%)$ & $219(34.5 \%)$ & \\
\hline N3 & $11(4.8 \%)$ & $50(7.2 \%)$ & & $10(4.4 \%)$ & $34(5.4 \%)$ & \\
\hline \multicolumn{7}{|l|}{ Clinical stage } \\
\hline III & $188(82.8 \%)$ & $533(76.3 \%)$ & 0.038 & $187(82.7 \%)$ & $519(81.9 \%)$ & 0.674 \\
\hline IV & $39(17.2 \%)$ & $166(23.7 \%)$ & & $38(17.3 \%)$ & $115(18.1 \%)$ & \\
\hline
\end{tabular}

Data represent the median (range) and $\mathrm{n}(\%)$, unless otherwise indicated. ^ $\chi^{2}$ test or Fisher's exact test. \& Mann-Whitney U-test.

Abbreviations: KPS, Karnofsky performance score. 


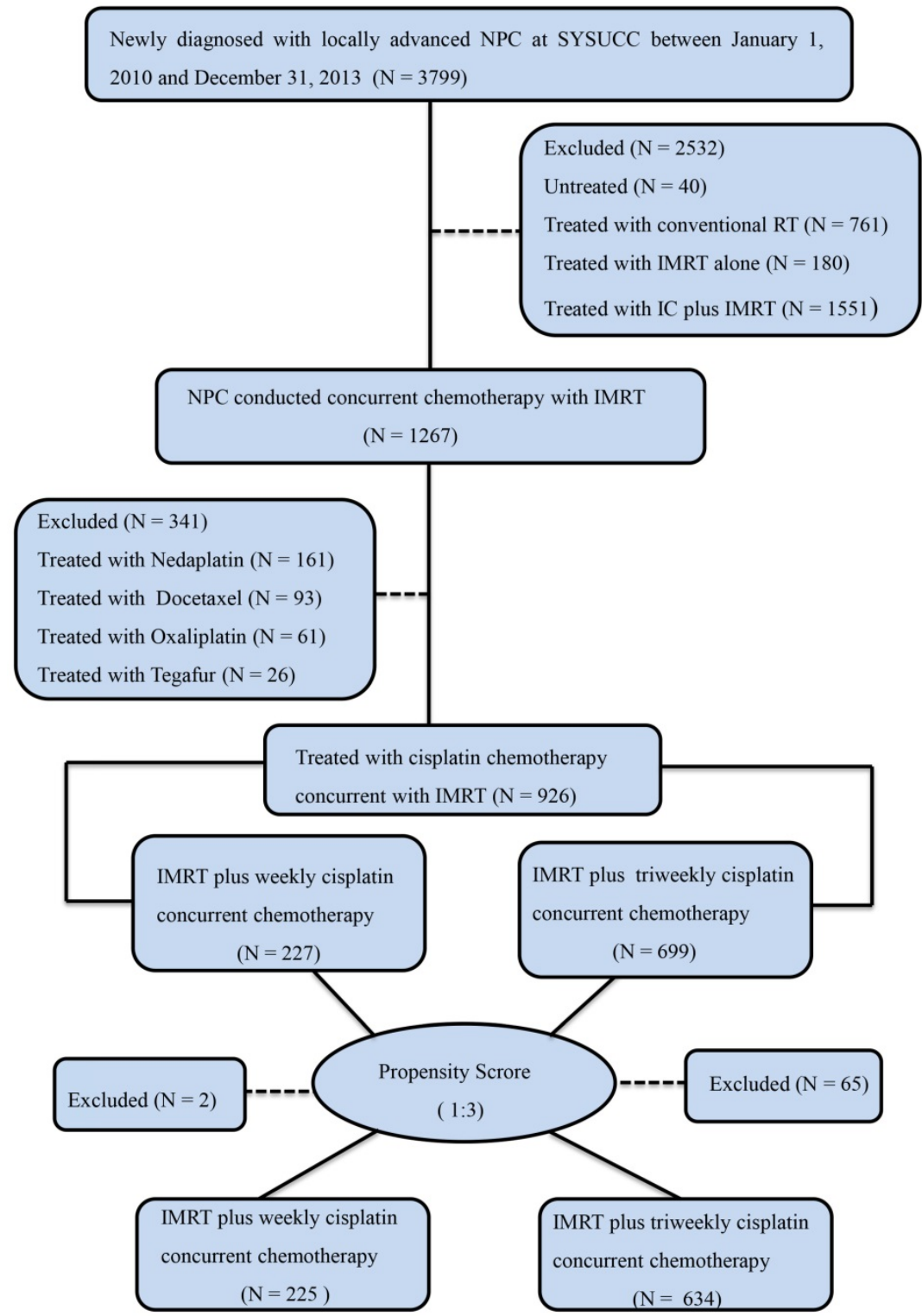

Figure 1: CONSORT flow diagram. Abbreviations: NPC: nasopharyngeal carcinoma; IMRT: intensity-modulated radiotherapy.

\section{Efficacy}

The median follow-up time was 51 months (range, 1 - 68 months) in the weekly group and 50 months (range, $1-69$ months) in the triweekly group. Patients in the weekly CDDP group (1-, 3- and 5-year DFS rate, $94 \%, 86 \%$ and $82 \%$, respectively) showed favourable DFS survival compared with patients in the triweekly group (1-, 3- and 5-year DFS rate, $89 \%$, $83 \%$ and $81 \%$, respectively), but a significant difference between these two groups was not observed $(P=.326$; Figure $2 \mathrm{~A})$. The risk of distant metastasis was lower among the patients treated with weekly CDDP than among those treated with triweekly CDDP (HR for DMFS, 0.45; 95\% CI, 0.21 $0.94 ; P=.028$; Figure $2 \mathrm{~B}$ ). Moreover, the $1-, 3$ - and 5 -year rates of DMFS achieved with weekly CDDP ( $97 \%, 97 \%$, and $96 \%$, respectively) were higher than those achieved with triweekly CDDP (94\%, 92\%, and $91 \%$, respectively). However, the LRRFS and OS were not significantly different between the two treatment groups (HR for LRRFS, 1.03; 95\% CI, $0.57-1.88$; $P=$ .932; Figure 2C; HR for OS, 1.11; 95\% CI, 0.65 - 1.90; $P$ $=.715$; Figure 2D). The 5-year LRRFS and OS rates were similar at $91 \%$ and $89 \%$, respectively, in the weekly group and $92 \%$ and $91 \%$, respectively, in the triweekly group.

\section{Safety}

The grade 3-4 systemic toxicities are displayed in Table 2. No treatment-related deaths were seen in either group. Of the 859 patients in the propensity score-matched cohort, the most common grade 3-4 haematological adverse event was leukopenia $(126 / 859,14.7 \%)$. The most commonly recorded grade 
A

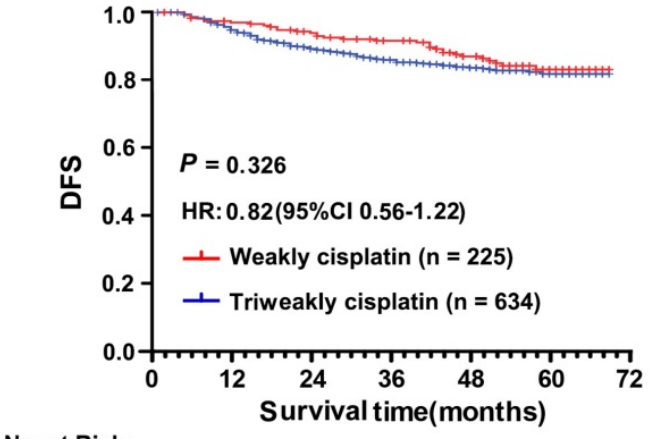

No. at Risk

$\begin{array}{lllllll}\text { Weakly } & 225 & 212 & 198 & 183 & 141 & 45 \\ \text { Triweakly } & 634 & 583 & 506 & 452 & 332 & 101\end{array}$

C

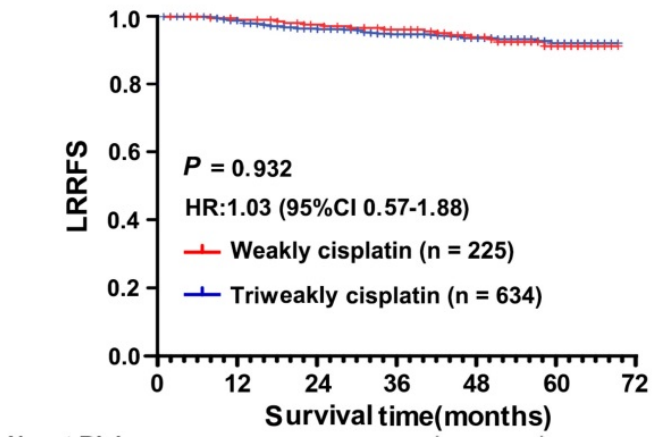

No. at Risk

$\begin{array}{lcccccc}\text { Weakly } & 225 & 214 & 203 & 188 & 146 & 46 \\ \text { Triweakly } & 634 & 596 & 528 & 473 & 342 & 103\end{array}$

B

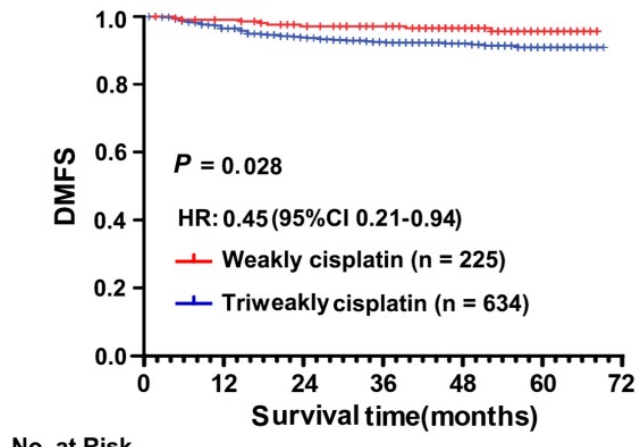

No. at Risk

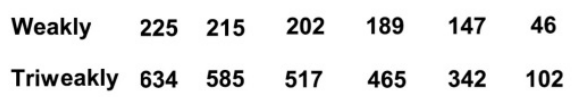

D

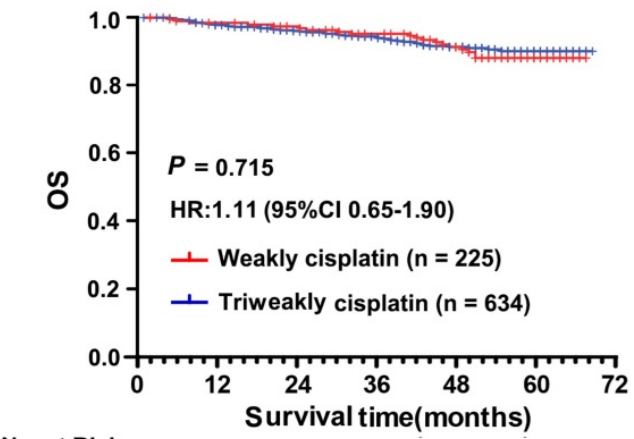

No. at Risk

$\begin{array}{llllllc}\text { Weakly } & 225 & 216 & 207 & 192 & 149 & 47 \\ \text { Triweakly } & 634 & 603 & 544 & 493 & 355 & 107\end{array}$

Figure 2: Comparison of survival according to weekly and triweekly concurrent cisplatin chemotherapy plus intensity-modulated radiotherapy regimen in the well-balanced cohort. (A) Disease-free survival. (B) Distant metastasis-free survival. (C) Loco-regional recurrence-free survival and (D) Overall survival. The hazard ratio $(\mathrm{HR})$ was calculated with the Cox proportional-hazards model; $P$ values were calculated with the log-rank test.

3-4 nonhaematological adverse event was mucositis $(147 / 859,17.1 \%)$. The weekly group showed significantly higher proportions of grade 3-4 thrombocytopenia than did the triweekly group (odds ratio [OR], 6.56; 95\% CI, $2.00-21.53 ; P=.002)$ but lower proportions of grade 3-4 mucositis (OR, 0.52; $95 \% \mathrm{CI}, 0.33-0.83 ; P=.006)$, nausea $(\mathrm{OR}, 0.20 ; 95 \% \mathrm{CI}$, $0.08-0.50 ; P=.001)$ and vomiting (OR, $0.21 ; 95 \% \mathrm{CI}$, $0.08-0.54 ; P=.001)$ than detected in the triweekly group.

Table 2. Acute adverse events in the propensity score-matched cohort of NPC patients

\begin{tabular}{|c|c|c|c|c|}
\hline Event & Triweekly $(\mathrm{N}=634)$ & Weekly $(\mathrm{N}=225)$ & OR $(95 \%$ CI) & $P$ \\
\hline \multicolumn{5}{|l|}{ Haematological } \\
\hline Anaemia & $6(0.9)$ & $4(1.8)$ & & 0.326 \\
\hline Thrombocytopenia & $4(0.6)$ & $9(4.0)$ & $6.56(2.00-21.53)$ & 0.002 \\
\hline Neutropenia & $36(5.7)$ & $18(8.0)$ & & 0.220 \\
\hline Leukopenia & 88 (13.9) & $38(16.8)$ & & 0.274 \\
\hline \multicolumn{5}{|l|}{ Non-haematological } \\
\hline Nephrotoxicity & $0(0.0)$ & $0(0.0)$ & & - \\
\hline Hepatoxicity & $5(0.8)$ & $2(0.9)$ & & 0.886 \\
\hline Nausea & 65 (10.3) & $5(2.2)$ & $0.20(0.08-0.50)$ & 0.001 \\
\hline
\end{tabular}

\begin{tabular}{lllll}
\hline Event & Triweekly (N=634) & Weekly (N=225) & OR (95\% CI) & $\boldsymbol{P}$ \\
\hline Vomiting & $61(9.6)$ & $5(2.2)$ & $0.21(0.08-0.54)$ & $\mathbf{0 . 0 0 1}$ \\
Diarrhea & $0(0.0)$ & $0(0.0)$ & & - \\
Skin reaction & $12(1.9)$ & $6(2.7)$ & & 0.488 \\
Mucositis & $122(19.2)$ & $25(11.1)$ & $0.52(0.33-0.83)$ & $\mathbf{0 . 0 0 6}$ \\
Weight loss & $6(0.9)$ & $2(0.9)$ & & 0.939 \\
\hline
\end{tabular}

Data represent the number of patients with events (\%). P values were calculated with the $\chi^{2}$ test.

\section{Multivariate analysis and subgroup analysis}

Univariate and multivariate analyses were conducted in the cohort of all 926 patients. Various prognostic parameters, including age $(\leq 45$ years $v s$. $>$ 45 years), sex, KPS status, T classification (T1-2 vs. T3 vs. T4), N classification (N0-1 vs. N2-3), clinical stage and concurrent chemotherapy regimen (weekly CDDP vs. triweekly CDDP), were included in the analysis. After adjusting for these parameters, significantly lower risks of distant metastasis (HR $0.43,95 \%$ CI $0.20-0.90, P=.026$ ) were found among patients treated with weekly CDDP than among patients treated with triweekly CDDP (Supplementary Table 1 and Table 3). Moreover, 
advanced $\mathrm{N}$ stage was a significant risk factor for DMFS (HR 1.89, 95\% CI $1.08-3.34, P=.027$ ) and OS (HR 1.72, 95\% CI $1.00-2.93, P=.048$ ). We further performed an interaction analysis, which demonstrated no significant interaction effect between treatment regimen (weekly vs. triweekly CDDP) and N classification from DFS, DMFS, LRRFS and OS (all $P>.05$, Table 4 ).

\section{Discussion}

The present study is the first propensity score analysis to evaluate the efficacy and acute toxicity of these two chemotherapy regimens using a large cohort (926 locally advanced NPC patients). PSM was used to balance the baseline characteristics between the groups, which allowed us to draw reliable conclusions. In both the pre-matched and matched cohorts, patients with locally advanced NPC treated with the weekly CDDP regimen achieved significantly improved DMFS but not DMS, LRRFS or OS.

Previous studies have detected the same insignificant results for DMS and OS when directly comparing the above concurrent chemotherapy regimens [15-17]. Considering the majority of treatment failures are due to distant metastasis, especially in patients with locally advanced stage disease [20, 21], our study used DMFS as the end point and detected a positive association with a weekly CDDP treatment schedule.

Table 3. Multivariate analysis in the unmatched group

\begin{tabular}{|c|c|c|c|c|c|c|c|c|c|c|c|c|}
\hline \multirow[t]{2}{*}{ Variable } & \multicolumn{3}{|c|}{ Disease-free survival } & \multicolumn{3}{|c|}{ Distant metastasis-free survival } & \multicolumn{3}{|c|}{ Loco-regional relapse-free survival } & \multicolumn{3}{|c|}{ Overall survival } \\
\hline & HR & CI ( $(\%)$ & $P$ & HR & CI (\%) & $P$ & HR & CI (\%) & $P$ & HR & CI (\%) & $P$ \\
\hline \multicolumn{13}{|l|}{ Sex } \\
\hline Female & Ref. & Ref. & & Ref. & Ref. & & Ref. & Ref. & & Ref. & Ref. & \\
\hline Male & 1.08 & $0.71-1.64$ & 0.712 & 1.63 & $0.83-3.22$ & 0.159 & 1.84 & $0.90-3.76$ & 0.094 & 0.98 & $0.56-1.69$ & 0.932 \\
\hline \multicolumn{13}{|l|}{ Age } \\
\hline$\leq 45$ & Ref. & Ref. & & Ref. & Ref. & & Ref. & Ref. & & Ref. & Ref. & \\
\hline$>45$ & 1.02 & $0.72-1.45$ & 0.908 & 0.74 & $0.44-1.24$ & 0.255 & 1.42 & $0.84-2.38$ & 0.188 & 2.29 & $1.40-3.73$ & 0.001 \\
\hline \multicolumn{13}{|l|}{ KPS } \\
\hline $70-80$ & Ref. & Ref. & & Ref. & Ref. & & Ref. & Ref. & & Ref. & Ref. & \\
\hline $90-100$ & 0.38 & $0.14-1.05$ & 0.061 & 0.25 & $0.08-0.83$ & 0.023 & 0.63 & $0.09-4.62$ & 0.650 & 0.34 & $0.10-1.11$ & 0.074 \\
\hline \multicolumn{13}{|c|}{ T classification } \\
\hline $\mathrm{T} 1-2$ & Ref. & Ref. & & Ref. & Ref. & & Ref. & Ref. & & Ref. & Ref. & \\
\hline $\mathrm{T} 3$ & 1.12 . & $0.62-2.02$ & 0.711 & 0.96 & $0.45-2.06$ & 0.915 & 1.33 & $0.55-3.18$ & 0.529 & 0.96 . & $0.47-1.93$ & 0.903 \\
\hline $\mathrm{T} 4$ & 1.53 & $0.60-3.92$ & 0.372 & 1.36 & $0.47-3.95$ & 0.572 & 4.78 & $0.64-35.53$ & 0.126 & 1.31 & $0.44-3.89$ & 0.625 \\
\hline \multicolumn{13}{|c|}{$\mathrm{N}$ classification } \\
\hline N0-1 & Ref. & Ref. & & Ref. & Ref. & & Ref. & Ref. & & Ref. & Ref. & \\
\hline N2-3 & 1.30 & $0.87-1.94$ & 0.202 & 1.89 & $1.08-3.34$ & 0.027 & 1.52 & $0.86-2.69$ & 0.151 & 1.72 & $1.00-2.93$ & 0.048 \\
\hline \multicolumn{13}{|c|}{ Clinical stage } \\
\hline III & Ref. & Ref. & & Ref. & Ref. & & Ref. & Ref. & & Ref. & Ref. & \\
\hline IV & 1.18 & $0.56-2.52$ & 0.663 & 1.90 & $0.80-4.49$ & 0.145 & 0.32 & $0.05-1.92$ & 0.211 & 1.71 & $0.73-4.01$ & 0.216 \\
\hline \multicolumn{13}{|l|}{ Treatment } \\
\hline Triweekly & Ref. & Ref. & & Ref. & Ref. & & Ref. & Ref. & & Ref. & Ref. & \\
\hline Weekly & 0.67 & $0.43-1.06$ & 0.084 & 0.43 & $0.20-0.90$ & 0.026 & 0.94 & $0.52-1.70$ & 0.836 & 1.10 & $0.64-1.86$ & 0.737 \\
\hline
\end{tabular}

Table 4. Interaction between treatment and other significant prognostic factors and its effect on disease-free survival, distant metastasis-free survival, loco-regional relapse-free survival, and overall survival

\begin{tabular}{|c|c|c|c|c|c|c|c|c|}
\hline & \multicolumn{2}{|l|}{ DFS } & \multicolumn{2}{|l|}{ DMFS } & \multicolumn{2}{|l|}{ LRRFS } & \multicolumn{2}{|l|}{ os } \\
\hline & Adjusted HR CI (\%) & $P$ & Adjusted HR CI (\%) & $P$ & Adjusted HR CI (\%) & $P$ & Adjusted HR CI (\%) & $P$ \\
\hline \multicolumn{9}{|c|}{ Treatment regimen status and Age } \\
\hline \multicolumn{9}{|l|}{ Treatment } \\
\hline Triweekly & Ref. & & Ref. & & Ref. & & Ref. & \\
\hline Weekly & $1.09(0.44-2.68)$ & 0.852 & $0.62(0.27-1.43)$ & 0.262 & $0.88(0.37-2.01)$ & 0.774 & $0.49(0.17-1.44)$ & 0.192 \\
\hline \multicolumn{9}{|l|}{ Age } \\
\hline$\leq 45$ & Ref. & & Ref. & & Ref. & & Ref. & \\
\hline$>45$ & $1.00(0.68-1.49)$ & 0.984 & $0.86(0.50-1.49)$ & 0.586 & $1.37(0.75-2.50)$ & 0.303 & $1.72(0.98-3.00)$ & 0.057 \\
\hline \multicolumn{9}{|c|}{ Interaction effect } \\
\hline Weekly * >45 & $1.09(0.44-2.68)$ & 0.852 & $0.22(0.03-1.94)$ & 0.174 & $1.13(0.35-3.70)$ & 0.839 & $3.25(0.93-11.32)$ & 0.065 \\
\hline \multicolumn{9}{|c|}{ Treatment regimen status and $\mathrm{N}$ classification } \\
\hline \multicolumn{9}{|c|}{ Treatment } \\
\hline Triweekly & Ref. & & Ref. & & Ref. & & Ref. & \\
\hline Weekly & $0.64(0.34-1.20)$ & 0.160 & $0.24(0.06-1.03)$ & 0.056 & $1.04(0.47-2.34)$ & 0.916 & $0.84(0.36-1.93)$ & 0.677 \\
\hline \multicolumn{9}{|l|}{ N classification } \\
\hline N0-1 & Ref. & & Ref. & & Ref. & & Ref. & \\
\hline N2-3 & $1.27(0.82-1.97)$ & 0.283 & $1.71(0.94-3.11)$ & 0.077 & $1.61(0.84-3.06)$ & 0.149 & $1.51(0.82-2.77)$ & 0.180 \\
\hline \multicolumn{9}{|c|}{ Interaction effect } \\
\hline Weekly *N2-3 & $1.12(0.46-2.73)$ & 0.808 & $2.33(0.43-12.67)$ & 0.326 & $0.80(0.25-2.61)$ & 0.713 & $1.61(0.55-4.76)$ & 0.389 \\
\hline
\end{tabular}


In the past decade, the potential benefit of using comparatively low doses of chemotherapy drugs on a more frequent schedule has gradually been appreciated [22]. Initially, it was reported that this type of regimen exerts effects exclusively by preventing angiogenesis [23]. Recent evidence revealed an additional mechanism, that the same overall dose administered as a low-dose regimen prevented carcinoma cell phenotypic conversion into stem-like tumour-initiating cells, thus enhancing treatment response and extending survival [24]. Intriguingly, the improvement in DMFS did not translate into an improvement in OS in the current study, which may be due to the $25 \%(14 / 56)$ of patients who developed distant metastasis and were still alive at the date of last follow-up. Therefore, further follow-up is needed to fully evaluate long-term survival.

Compared with conventional radiotherapy, IMRT has the advantage of improving target coverage and applying a more accurate dose to the tumour target while sparing normal organs [25-27]. Due to recent advances in the radiation techniques of IMRT, a $90 \%$ local control rate for NPC treated with IMRT plus chemotherapy has been reported, even for patients with locally advanced disease [28]. In our study, the 5 -year LRRFS was $91 \%$ in the weekly group and $92 \%$ in the triweekly group. The above favourable results of LRRFS in both groups might have narrowed any potential therapeutic gain of concurrent chemotherapy for local control.

The cumulative dose intensity of cisplatin has been reported to be an important prognostic factor in NPC patients treated with CCRT regimens [29]. However, the mean cumulative cisplatin dose (weekly vs. triweekly, $229.2 \mathrm{mg} / \mathrm{m}^{2}$ vs. $228.0 \mathrm{mg} / \mathrm{m}^{2}$ ) was similar between groups in our study. Although lower compliance rates of concurrent cisplatin chemotherapy were detected when compared with previous trials $[15,17]$, it should be noted that the majority of patients received at least five cycles of CDDP in the weekly group (92\%) and two cycles of CDDP in the triweekly group (97\%). In our cancer centre, weekly cisplatin at a dose of $40 \mathrm{mg} / \mathrm{m}^{2}$ is mainly delivered to outpatients, while $100 \mathrm{mg} / \mathrm{m}^{2}$ cisplatin triweekly concurrently with IMRT regimen is mostly administered to hospitalized patients [16]. Poor compliance by outpatients and insufficient hospital beds often lead to the postponement of concurrent chemotherapy and patients skipping the final cycle of chemotherapy.

The concept of lower-dose cisplatin, given on a weekly basis concurrent with radiotherapy, is based on the hypothesis that an intermediate cisplatin dose with frequently administered will decrease the toxicity profile while maintaining efficacy. A previous randomized phase III trial confirmed that weekly cisplatin concurrent with radiotherapy conferred a survival benefit compared with radiotherapy alone [12]. However, the difference in the toxicity profile between the two concurrent chemotherapy schedules is controversial. Ho et al. found that the incidence of grade 3-4 toxic events of any type was not decreased in locally advanced patients treated with the weekly regimen[30]. Conversely, Tao et al. demonstrated a slightly lower incidence of grade 3-4 dysphagia and gastrointestinal reactions with a weekly regimen (dysphagia, 4.1\%; gastrointestinal reactions, $0.0 \%$; respectively) than with a triweekly regimen (dysphagia, 6.2\%; gastrointestinal reactions, 3.7\%; respectively), but this difference was not significant [16]. Based on a large cohort, our study found a significant difference in severe toxicity with a higher incidence of thrombocytopenia, whereas there was a lower incidence of grade 3-4 gastrointestinal reactions (nausea and vomiting) and mucositis with the weekly CDDP regimen. Hence, clinicians should adopt aggressive action such as adequate monitoring of blood counts and oral care to avoid any severe toxicity depending on the choice of concurrent chemotherapy treatment regimen.

Our study has several limitations. Although PSM analysis was conducted to minimize the impact of observed confounders [31,32], it is unclear whether other confounding factors still exist. Quantification of plasma Epstein-Barr virus (EBV) DNA has been demonstrated to be useful for predicting the survival outcome in advanced NPC patients [33]. However, the EBV DNA load was not included in our study because standardization of the assay remained unresolved before the patients enrolled in the study. Moreover, the present study was a single-centre retrospective study, and further multi-centre prospective randomized studies are needed.

\section{Conclusions}

IMRT concurrent with a weekly cisplatin regimen significantly improved DMFS but not DMS, LRRFS or OS than triweekly cisplatin regimen in patients with locally advanced NPC. Differences in the selected acute toxicities were also observed between the two groups. This study will provide evidence for clinicians to choose the appropriate strategy for locally advanced NPC patients and inform the design of future prospective studies.

\section{Abbreviations}

IMRT: intensity-modulated radiotherapy; NPC: nasopharyngeal carcinoma; CDDP: cisplatin-based concomitant chemo-radiotherapy; DMFS: distant 
metastasis-free survival; LRRFS: loco-regional relapse-free survival; OS: overall survival; DFS: disease-free survival; CTCAE: Common Terminology Criteria for Adverse Events; KPS: Karnofsky performance status.

\section{Supplementary Material}

Supplementary figure and table. http://www.jcancer.org/v09p3447s1.pdf

\section{Acknowledgements}

This work was supported by a grant from the National Natural Science Foundation for Young Scholar of China (81402560), the National Natural Science Foundation of China (81572865, 81773110 and 81472387), and the Guangdong Province Science and Technology Plan Project (2013B021800063).

\section{Ethics Committee Approval and Patient Consent}

The use of clinical data were reviewed and approved by the Institutional Ethics Committee of the Sun Yat-sen University Cancer Center (China). As a retrospective study, the informed consent was verbal.

\section{Competing Interests}

The authors have declared that no competing interest exists.

\section{References}

1. Chang ET, Adami HO. The enigmatic epidemiology of nasopharyngeal carcinoma. Cancer epidemiology, biomarkers \& prevention : a publication of the American Association for Cancer Research, cosponsored by the American Society of Preventive Oncology. 2006; 15: 1765-77.

2. Zhang GY, Liu LZ, Wei WH, Deng YM, Li YZ, Liu XW. Radiologic criteria of retropharyngeal lymph node metastasis in nasopharyngeal carcinoma treated with radiation therapy. Radiology. 2010; 255: 605-12.

3. Liu L, Liang S, Li L, Mao Y, Tang L, Tian L, et al. Prognostic impact of magnetic resonance imaging-detected cranial nerve involvement in nasopharyngeal carcinoma. Cancer. 2009; 115: 1995-2003.

4. Chen L, Mao YP, Xie FY, Liu LZ, Sun Y, Tian L, et al. The seventh edition of the UICC/AJCC staging system for nasopharyngeal carcinoma is prognostically useful for patients treated with intensity-modulated radiotherapy from an endemic area in China. Radiotherapy and oncology: journal of the European Society for Therapeutic Radiology and Oncology. 2012; 104: 331-7.

5. Afqir S, Ismaili N, Errihani H. Concurrent chemoradiotherapy in the management of advanced nasopharyngeal carcinoma: current status. Journal of cancer research and therapeutics. 2009; 5: 3-7.

6. Teo PM, Kwan WH, Lee WY, Leung SF, Johnson PJ. Prognosticators determining survival subsequent to distant metastasis from nasopharyngeal carcinoma Cancer 1996; 77: 2423-31.

7. Geara FB, Sanguineti G, Tucker SL, Garden AS, Ang KK, Morrison WH, et al. Carcinoma of the nasopharynx treated by radiotherapy alone: determinants of distant metastasis and survival. Radiotherapy and oncology: journal of the European Society for Therapeutic Radiology and Oncology. 1997; 43: 53-61.

8. Hui EP, Leung SF, Au JS, Zee B, Tung S, Chua D, et al. Lung metastasis alone in nasopharyngeal carcinoma: a relatively favorable prognostic group. A study by the Hong Kong Nasopharyngeal Carcinoma Study Group. Cancer. 2004; 101: 300-6.

9. Al-Sarraf M, LeBlanc M, Giri PG, Fu KK, Cooper J, Vuong T, et al. Chemoradiotherapy versus radiotherapy in patients with advanced nasopharyngeal cancer: phase III randomized Intergroup study 0099. Journal of clinical oncology: official journal of the American Society of Clinical Oncology. 1998; 16: 1310-7.

10. Lin JC, Jan JS, Hsu CY, Liang WM, Jiang RS, Wang WY. Phase III study of concurrent chemoradiotherapy versus radiotherapy alone for advanced nasopharyngeal carcinoma: positive effect on overall and progression-free survival. Journal of clinical oncology : official journal of the American Society of Clinical Oncology. 2003; 21: 631-7.

11. Lee AW, Tung SY, Chua DT, Ngan RK, Chappell R, Tung R, et al. Randomized trial of radiotherapy plus concurrent-adjuvant chemotherapy vs radiotherapy alone for regionally advanced nasopharyngeal carcinoma. Journal of the National Cancer Institute. 2010; 102: 1188-98.

12. Chan AT, Leung SF, Ngan RK, Teo PM, Lau WH, Kwan WH, et al. Overall survival after concurrent cisplatin-radiotherapy compared with radiotherapy alone in locoregionally advanced nasopharyngeal carcinoma. Journal of the National Cancer Institute. 2005; 97: 536-9.

13. Lee AW, Ma BB, Ng WT, Chan AT. Management of Nasopharyngeal Carcinoma: Current Practice and Future Perspective. Journal of clinical oncology: official journal of the American Society of Clinical Oncology. 2015; 33: 3356-64.

14. National Comprehensive Cancer Network. NCCN Guidelines: Head and neck cancers; Version 2. 2013.

15. Jagdis A, Laskin J, Hao D, Hay J, Wu J, Ho C. Dose delivery analysis of weekly versus 3-weekly cisplatin concurrent with radiation therapy for locally advanced nasopharyngeal carcinoma (NPC). American journal of clinical oncology. 2014; 37: 63-9.

16. Tao CJ, Lin L, Zhou GQ, Tang LL, Chen L, Mao YP, et al. Comparison of long-term survival and toxicity of cisplatin delivered weekly versus every three weeks concurrently with intensity-modulated radiotherapy in nasopharyngeal carcinoma. PloS one. 2014; 9: e110765.

17. Lee JY, Sun JM, Oh DR, Lim SH, Goo J, Lee SH, et al. Comparison of weekly versus triweekly cisplatin delivered concurrently with radiation therapy in patients with locally advanced nasopharyngeal cancer: A multicenter randomized phase II trial (KCSG-HN10-02). Radiotherapy and oncology : journal of the European Society for Therapeutic Radiology and Oncology. 2016; 118: 244-50.

18. Peng $\mathrm{G}$, Wang $\mathrm{T}$, Yang $\mathrm{KY}$, Zhang $\mathrm{S}$, Zhang $\mathrm{T}$, Li $\mathrm{O}$, et al. A prospective, randomized study comparing outcomes and toxicities of intensity-modulated radiotherapy vs. conventional two-dimensional radiotherapy for the treatment of nasopharyngeal carcinoma. Radiotherapy and oncology : journal of the European Society for Therapeutic Radiology and Oncology. 2012; 104: 286-93.

19. Zhao C, Han F, Lu LX, Huang SM, Lin CG, Deng XW, et al. [Intensity modulated radiotherapy for local-regional advanced nasopharyngeal carcinoma]. Ai zheng = Aizheng $=$ Chinese journal of cancer. 2004; 23: 1532-7.

20. Lee AW, Sze WM, Au JS, Leung SF, Leung TW, Chua DT, et al. Treatment results for nasopharyngeal carcinoma in the modern era: the Hong Kong experience. International journal of radiation oncology, biology, physics. 2005; 61: 1107-16.

21. Ma BB, Hui EP, Chan AT. Systemic approach to improving treatment outcome in nasopharyngeal carcinoma: current and future directions. Cancer science. 2008; 99: 1311-8

22. Loven D, Hasnis E, Bertolini F, Shaked Y. Low-dose metronomic chemotherapy: from past experience to new paradigms in the treatment of cancer. Drug discovery today. 2013; 18: 193-201.

23. Pasquier E, Kavallaris M, Andre N. Metronomic chemotherapy: new rationale for new directions. Nature reviews Clinical oncology. 2010; 7: 455-65.

24. Chan TS, Hsu CC, Pai VC, Liao WY, Huang SS, Tan KT, et al. Metronomic chemotherapy prevents therapy-induced stromal activation and induction of tumor-initiating cells. The Journal of experimental medicine. 2016; 213: 2967-88.

25. Kam MK, Teo PM, Chau RM, Cheung KY, Choi PH, Kwan WH, et al. Treatment of nasopharyngeal carcinoma with intensity-modulated radiotherapy: the Hong Kong experience. International journal of radiation oncology, biology, physics. 2004; 60: 1440-50.

26. Lee N, Xia P, Quivey JM, Sultanem K, Poon I, Akazawa C, et al. Intensity-modulated radiotherapy in the treatment of nasopharyngeal carcinoma: an update of the UCSF experience. International journal of radiation oncology, biology, physics. 2002; 53: 12-22.

27. Cheung K. Intensity modulated radiotherapy: advantages, limitations and future developments. Biomedical imaging and intervention journal. 2006; 2: e19.

28. Lee N, Harris J, Garden AS, Straube W, Glisson B, Xia P, et al. Intensity-modulated radiation therapy with or without chemotherapy for nasopharyngeal carcinoma: radiation therapy oncology group phase II trial 0225. Journal of clinical oncology: official journal of the American Society of Clinical Oncology. 2009; 27: 3684-90.

29. Loong HH, Ma BB, Leung SF, Mo F, Hui EP, Kam MK, et al. Prognostic significance of the total dose of cisplatin administered during concurrent chemoradiotherapy in patients with locoregionally advanced nasopharyngeal carcinoma. Radiotherapy and oncology: journal of the European Society for Therapeutic Radiology and Oncology. 2012; 104: 300-4.

30. Ho KF, Swindell R, Brammer CV. Dose intensity comparison between weekly and 3-weekly Cisplatin delivered concurrently with radical radiotherapy for head and neck cancer: a retrospective comparison from New Cross Hospital, Wolverhampton, UK. Acta oncologica. 2008; 47: 1513-8.

31. Tang LQ, Li CF, Li J, Chen WH, Chen QY, Yuan LX, et al. Establishment and Validation of Prognostic Nomograms for Endemic Nasopharyngeal Carcinoma. Journal of the National Cancer Institute. 2016; 108

32. You R, Hua YJ, Liu YP, Yang Q, Zhang YN, Li JB, et al. Concurrent Chemoradiotherapy with or without Anti-EGFR-Targeted Treatment for Stage 
II-IVb Nasopharyngeal Carcinoma: Retrospective Analysis with a Large Cohort and Long Follow-up. Theranostics. 2017; 7: 2314-24.

33. Lin JC, Wang WY, Chen KY, Wei YH, Liang WM, Jan JS, et al. Quantification

of plasma Epstein-Barr virus DNA in patients with advanced nasopharyngeal carcinoma. The New England journal of medicine. 2004; 350: 2461-70. 